\title{
Editorial: Topical Collection to "Reading Terrestrial Planet Evolution in Isotopes and Element Measurements"
}

\author{
Helmut Lammer ${ }^{1} \cdot$ Bernard Marty $^{2} \cdot$ Aubrey L. Zerkle $^{3}$. Manuel Scherf ${ }^{1}$. \\ Hugh O'Neill ${ }^{4}$. Michel Blanc ${ }^{5}$. Thorsten Kleine ${ }^{6}$
}

Received: 6 May 2021 / Accepted: 6 May 2021 / Published online: 17 May 2021

(c) The Author(s), under exclusive licence to Springer Nature B.V. 2021

Terrestrial planets evolve from primitive material of which remnants are now found in primitive meteorites called chondrites that originated from parent bodies that did not go through the cycle of melting and differentiation. Two broad categories have been defined based on their elemental and isotopic abundances, the carbonaceous chondrites (CC) and the noncarbonaceous chondrites (NC). Using geochemical observations of noble gases and other major volatiles, one can determine what the present-day inventory tells us on its initial building blocks, their sources, accretion processes and the early differentiation of planetary bodies such as the Earth. While many of the present-day volatile reservoirs have chondritic isotopic ratios, their relative abundances are certainly not chondritic, suggesting volatile losses from planetary embryos and growing proto- and early planets during and after their accretion phases. The interdisciplinary research fields discussed contain different processes that produce chemical compositional changes after the solar nebula/chondrite stage. These, for instance, contain collisional erosion, post-nebula volatilization and the escape of noble gases and volatile elements that were dragged by hydrodynamically escaping hydrogen atoms originating from captured primordial atmospheres or magma ocean-related catastrophic outgassed steam atmospheres.

The above-mentioned losses and their efficiencies are dependent on the disk lifetime, the X-ray, and EUV flux evolution of a system's young host star and the accretion history of planets in general. Isotopic and elemental ratios can also be used for constraining the

Reading Terrestrial Planet Evolution in Isotopes and Element Measurements

Edited by Helmut Lammer, Bernard Marty, Aubrey L. Zerkle, Michel Blanc, Hugh O’Neill and Thorsten Kleine

\section{H. Lammer}

Space Research Institute, Austrian Academy of Sciences, Schmiedlstr. 6, 8042 Graz, Austria

2 CNRS, CRPG, Université de Lorraine, 54000 Nancy, France

3 School of Earth and Environmental Sciences and Centre for Exoplanet Science, University of St. Andrews, St. Andrews, UK

4 College of Physical \& Mathematical Sciences Research School of Earth Sciences, Australian National University Canberra, Canberra, Australia

5 Institut de Recherche en Astrophysique et Planétologie, IRAP, Toulouse, France

6 Institut für Planetologie, Universität Münster, Münster, Germany 
timescale of accretion, the volatile delivery and the origin of the Moon. The amount of radioactive heat producing elements (i.e., U, Th, K) for instance determines the planet's subsequent tectonic regimes and, therefore, its possible evolution to an Earth-like habitat.

Since the discovery of small rocky exoplanets, many speculations on "Earth-like habitats" buzz around within the exoplanet research community and hence the media and the public. That is why it is very important to understand how Earth obtained its main atmospheric species, $\mathrm{N}_{2}$ and, later on, $\mathrm{O}_{2}$. Terrestrial planet evolution in relation to the formation of modern plate tectonics and the influence of life on the origin of Earth's $\mathrm{N}_{2}$-dominated atmosphere, which can be seen as an atmospheric geo-signature and together with $\mathrm{O}_{2}$ as a geobiosignature, is also addressed in particular reviews of this topical collection. Future space missions that are important for enhancing our understanding on the evolution of terrestrial planets, including exoplanetary Earth-like habitats, are also discussed. Two special articles, one related to the deep nitrogen and carbon cycles on Earth and a second one, which summarizes the up-to-date data on the relative atomic Solar System abundances, mass fractions, atomic masses of the elements and their isotopes, the composition of the solar photosphere and of the major chondritic meteorite groups finish the collection.

\section{The Sun Through Time}

This topical collection starts with a review article by $M$. Güdel on the importance and role of the young Sun and its activity evolution through time by forcing the evolution of terrestrial planets, especially their atmospheres. The review focuses on the activity evolution of Sunlike G-type stars and discusses direct and indirect observations related to the spin down, of $\mathrm{X}$-ray activities and wind mass loss rates as a function of stellar mass and ages. From these observations, one finds that G-type stars that were born with different rotational periods, from slow, via moderate to fast rotators, have initial EUV fluxes of about 400 to 500 times higher than the present-day Sun during their saturation phase. This phase, however, lasts much longer for fast rotators, than for moderate, and particularly slow rotators. After these saturation phases, the winds and the stellar EUV radiation decline and converge after about 1 billion years (Gyr) after the G star's origin. These relations are particularly important for studying atmospheric evolution since the absorption of the host star's EUV flux heats the upper atmosphere, thereby enhancing thermal and non-thermal escape of particles in many complex ways. EUV-driven atmospheric loss processes also modify volatile element abundances and the ratios of atmospheric isotopes mass-dependently over time, which can tell us something about the rotational history of the planet's host star such as our Sun.

\section{Chemical and Isotopic Evolution of the Early Solar System}

The significance of the chemical and isotopic compositions of early planetary building blocks in the protoplanetary disk, is reviewed by Bermingham et al. The authors examine constraints on the chemical evolution of the disk provided by elemental and nucleosynthetic isotopic compositions of meteorites and discuss if and how the compositional evolution of the disk to the planetary system can be derived by synthesizing astrophysical models and datasets from meteorites that directly sample the disk. A fundamental question regarding the formation of the planets in the solar system concerns the nature and location of the noncarbonaceous (NC) and carbonaceous chondrite (CC) reservoirs, and possible interactions between these reservoirs. Evidence that $\mathrm{CC}$ meteorites formed in the outer protoplanetary 
disk beyond Jupiter's orbit, the contribution of $\mathrm{NC}$ and $\mathrm{CC}$ reservoirs to the formation of the Earth and its water, and challenges associated with the practice of inferring (radial) accretion locations and movement within the disk based on the volatile element and nucleosynthetic isotope compositions of meteorites are also discussed. Because several reviews of this topical collection have a strong focus on $\mathrm{N}_{2}$ and the evolution to Earth's $\mathrm{N}_{2}-\mathrm{O}_{2}$ dominated present-day atmosphere, this review also discusses the origin and reservoirs of nitrogen based on isotopic constraints provided by carbonaceous and enstatite chondrites. Overall, Earth's hydrogen, oxygen, nitrogen, and nucleosynthetic isotope signatures require building blocks from both the $\mathrm{NC}$ and $\mathrm{CC}$ reservoirs.

\section{Formation of Venus, Earth and Mars: Constrained by Isotopes}

The third review by Lammer et al. first discusses the current state of knowledge of terrestrial planet formation by reviewing different planet formation models based on the classical, annulus, grand-tack, depleted disk, pebble accretion and early instability hypotheses. A very new aspect of this review article is that the authors summarized the current state of knowledge of terrestrial planet formation not only from the aspects of different planet formation models but also from isotopic data of ${ }^{182} \mathrm{Hf}-{ }^{182} \mathrm{~W}$ geochronology, U-Pb chronology, lithophile-siderophile elements, ${ }^{48} \mathrm{Ca} /{ }^{44} \mathrm{Ca}$ isotope samples from planetary building blocks, recent reproduction attempts from $\mathrm{Ar}$ and $\mathrm{Ne}$ isotope ratios in Venus' and Earth's atmospheres, the expected solar ${ }^{3} \mathrm{He}$ abundance in Earth's deep mantle and Earth's D/H sea water ratios. By discussing the analysis of these different isotope data and chronometrical dating methods, one can conclude that terrestrial planets in the Solar System favored most likely fast accretion scenarios with $\approx 80 \%$ of Earth's mass most likely accreted within $\approx 10-30 \mathrm{Myr}$, while Venus could have even accreted up to its final mass until the end of the disk lifetime. For classical terrestrial planet formation models, however, it is a challenge to accrete large planetary embryos so fast from the dusty materials within the typical disk lifetimes. The authors of this article, therefore, conclude that pebble accretion and streaming instabilities could solve this timescale controversy. The later stage of accretion can be explained well with the Grand-Tack model and partly also by the annulus and depleted disk models. More studies, however, are needed to homogenise these studies with data that are provided by chemical, chronological, isotopical and dynamical constraints.

\section{Geochemical Constraints on the Origin of the Moon and the Preservation of Ancient Heterogeneities}

The latest hypotheses on the origin of the Moon by addressing geochemical constraints and ancient heterogeneities are discussed in the review by Lock et al. Although the giant impact theory, with a big impactor named Theia vaporizing much of the early Earth, is the accepted mainstream formation model since about 40 years, details of the Moon's formation remain not well understood until today. Studies show that giant impacts with angular momenta as low as necessary for the Moon-forming event would be a rare one, related to the lowest energy accretionary impact scenario between two massive bodies involved. Therefore, highenergy impacts are now proposed in addition since they provide new environments for the formation of the Moon by reproducing a new class of bodies exceeding the corotation limit, called "synestias", a dynamical, donut shaped, structure of vaporized rock. Regarding the timing of the Moon-forming event, various studies that analyzed isotopic measurements of 
Lunar, terrestrial and meteoritic samples that constrain a most likely formation age between $\approx 50-200$ Myr after the origin of the solar system, with most dating systems favoring ages closer to the younger period. One can argue that the isotopic similarity between the Earth and Moon requires that either the proto-Earth or its large impactor Theia had a very similar isotopic composition, or that the efficiency of mixing during the collision was high. However, the impact between proto-Earth and Theia should not have homogenized early Earth's whole mantle since isotopic signatures of pre-impact heterogeneities are preserved.

\section{On the Distribution and Variation of Radioactive Heat Producing Elements Within Meteorites, the Earth, and Planets}

The relevance of the distribution and variation of radioactive heat producing elements (HPEs) within meteorites and terrestrial planets as a main factor in controlling a rocky planet's thermal evolution, tectonics, and even its likelihood for habitability is reviewed by $O$ 'Neill et al. This type of research and findings are often ignored in habitability studies within the Astrobiology/exoplanet community. The state of knowledge of various HPEs in planetary building blocks, terrestrial planets, and in present Earth models from emerging constraints, and their role related to the tectonic history is summarized. The primordial sources of heat production within terrestrial planets such as the Earth include heat from the accretion process, core formation and heating due to ancient radioactive isotopes in the planet's history. The authors also present a heuristic way to investigate the sensitivity of Earth's current tectonic mode as an example for rocky planets in general to the planet's HPE inventory. They point out that the specifics of tectonic transitions are subject to ongoing debates in the geodynamic research community. There is, however, a common denominator; the internal heating rates of terrestrial planets and their HPE budget are fundamental in controlling the evolutionary paths to Earth-like habitats. Because HPEs and their related processes affect volcanic outgassing and tectonics-related in-gassing, the major atmospheric species associated to these processes, $\mathrm{CO}_{2}, \mathrm{SO}_{2}$ and $\mathrm{H}_{2} \mathrm{O}$, may be detectable by future spectroscopic measurements on terrestrial exoplanets.

\section{Loss and Fractionation of Noble Gas Isotopes and Moderately Volatile Elements from Planetary Embryos and Early Venus, Earth and Mars}

The latest knowledge on how various atmospheric escape processes can fractionate noble gas isotopes and moderately volatile rock-forming elements that populate accreting and primordial atmospheres as well as catastrophically outgassed steam atmospheres is reviewed by Lammer et al. The authors review and summarize the measured isotopes and their ratios in the atmospheres of Venus, Earth, and Mars. Especially for Venus' case, it is pointed out that several isotope measurements have large error bars or were not measured by previous spacecraft, therefore, it is highly recommended that new precise measurements should be carried out in future missions. Recent discoveries of $\mathrm{H}_{2}$-dominated primordial atmospheres on most detected low mass exoplanets with known radii and masses are also discussed. Most of these exoplanets have very low densities which indicate that the transit radius is not related to the rocky surface of these bodies, and only extended $\mathrm{H}_{2}$-dominated gas envelopes can explain the observations. From these discoveries, one can expect that many low mass 
exoplanets may have Earth-like density cores but a large number of them still host an $\mathrm{H}_{2}$ dominated primordial atmosphere that was captured from the circumstellar disk - favoring fast accretion scenarios during the lifetime of the gas disk. Finally, it is pointed out that a complex interplay between the young Sun's EUV flux, the accreted protoplanetary mass within the disk lifetime, a possible accumulated primordial $\mathrm{H}_{2}$-dominated atmosphere, the impacting bodies that were involved in the accretion before and after disk dispersal, as well as the depletion of volatile elements from planetary embryos and the final protoplanets eventually led to today's measured ratios.

\section{Nitrogen Atmospheres of the Icy Bodies in the Solar System}

The current knowledge on the origin and evolution of $\mathrm{N}_{2}$-dominated atmospheres on icy bodies such as Titan, Triton and Pluto is reviewed by Scherf et al. The authors point out that an important information for understanding the origin and evolution of these atmospheres can be found in various atmospheric isotopic signatures such as ${ }^{14} \mathrm{~N} /{ }^{15} \mathrm{~N},{ }^{12} \mathrm{C} /{ }^{13} \mathrm{C}$ and $\mathrm{Ar} / \mathrm{N}$ ratios. These isotope ratios in $\mathrm{N}_{2}$-dominated atmospheres serve as a footprint of the building blocks from which Titan, Triton and Pluto originated and by studying different fractionation processes one can figure out how their atmospheres evolved during their entire evolution. Studies that find that hydrodynamic escape, sputtering and Jeans escape cannot explain the strong enrichment of Titan's atmospheric ${ }^{15} \mathrm{~N}$ isotopes over its history if it would have the same origin as the Earth's, which is believed to be chondritic are addressed. Instead, the outgassing of $\mathrm{N}_{2}$ produced from cometary $\mathrm{NH}_{3}$ might have played a crucial role in Titan's history pointing to an endogenic origin, which is also backed by the existence of outgassed radiogenic ${ }^{40} \mathrm{Ar}$ isotopes in the atmosphere. The origin and evolution of the $\mathrm{N}_{2}$-dominated atmospheres of Neptune's large satellite Triton and the dwarf planet Pluto, even after the New Horizon flyby, remain unclear, although it might be likely that these atmospheres originated in a similar way as Titan's. This review also includes a discussion on the diverse nitrogen isotope measurement techniques since an accurate retrieval of isotopic ratios depends on different model and measurement assumptions.

\section{Perspectives on Atmospheric Evolution from Xe and Nitrogen Isotopes on Earth, Mars and Venus}

The latest knowledge on the evolution of Earth's, Venus' and Mars' atmospheres that can be constrained by Xe and nitrogen isotopes is reviewed by Avice and Marty. A major finding for the Earth's paleo-atmosphere is that xenon, the heaviest noble gas, is fractionated in Earth's atmosphere. They point out that the isotopic fractionation of Xe did not take place during or just after Earth's formation but occurred over Gyr timescales. Escape models that try to explain the Xe paradox and summarize the most likely ones, which is dragging of $\mathrm{Xe}^{+}$ions by EUV-powered hydrodynamic escape of $\mathrm{H}^{+}$atoms during the Archean, and the preferential trapping of heavier $\mathrm{Xe}^{+}$isotopes into organic hazes, which are believed to be abundant in the Archean atmosphere are reviewed and discussed. Analyses of Martian meteorites show that the isotopic fractionation and escape of Xe at Mars happened earlier (> $4 \mathrm{Ga}$ ago) compared to Earth. For Venus, none of the past space missions managed to detect $\mathrm{Xe}$ in its atmosphere. Besides Xe, atmospheric nitrogen and its isotopes can also be used as a tracer of past atmospheric pressure and escape. The authors themselves, for instance, found from data correlations of $\mathrm{N}_{2} /{ }^{36} \mathrm{Ar}$ vs. ${ }^{40} \mathrm{Ar} /{ }^{36} \mathrm{Ar}$ ratios that the partial pressure of 
atmospheric $\mathrm{N}_{2}$ was in the range of $0.5-1.0$ bar $\geq 3.0 \mathrm{Gyr}$ and most likely lower than the modern one at 3.3 Gyr. Contrary to Earth's, Mars' atmosphere is strongly enriched in the heavy ${ }^{15} \mathrm{~N}$ isotope, which is an indication for atmospheric escape of nitrogen during the past $\approx 4$ Gyr. The isotopic composition of $\mathrm{N}_{2}$ in Venus' atmosphere remains largely unknown with estimates of an Earth-like value within an uncertainty of $\approx 20 \%$.

\section{Mission to Planet Earth: The First Two Billion Years}

The evolution of early Earth with its focus on the first 2 Gyr after the origin of our home planet is reviewed by Stïeken et al. They identify five crucial main phases in early Earth's evolution: i) the redox state and volatile content of Earth's building blocks; ii) the chemical properties of the resulting degassed mantle which are important in its ability to recycle volatiles and nutrients via plate tectonics; iii) the emergence of life and life's capabilities to effect Earth's atmosphere (e.g. biological $\mathrm{CO}_{2}$ and $\mathrm{N}_{2}$ fixation, oxygenic photosynthesis, etc.); iv) the young Sun's higher EUV flux; and v) evidence of strong photochemical effects on Earth's sulfur (S) cycle, preserved in the form of mass-independent $\mathrm{S}$ isotope fractionation, and potentially linked to fractionation in organic C-isotopes. Regarding Earth's atmospheric $\mathrm{N}_{2}$ build-up, the latest hypotheses and evidence backed by isotope and ancient rock-related air pressure studies are discussed. It is shown that photochemical and geological processes set the stage for habitable conditions and how the origin of life likely had a significant impact on volatile inventories and the atmosphere with major implications for global climate and habitability. Relevant for the search of $\mathrm{N}_{2}$ atmospheres on terrestrial exoplanets, atmospheric biosignatures on the early Earth are also addressed and explain why $\mathrm{N}_{2}-\mathrm{O}_{2}$-dominated atmospheres most likely represent geo-biosignatures. This review shows very well that early Earth itself presents an exoplanet analogue that can be investigated through the existing rock record.

\section{The Isotopic Imprint of Life on an Evolving Planet}

The biological information recorded by stable isotopic compositions observed on Earth in the context of evolving metabolisms on an evolving planet is discussed by the review of Lloyd et al. They discuss the isotope biogeochemistry of the biologically essential elements $\mathrm{C}, \mathrm{N}$ and $\mathrm{S}$, and how these elements are distributed on the modern and early Earth, which is the closest analogue for an extraterrestrial Earth-like habitat. They describe how lifeforms exist in thermodynamic disequilibrium with their environment, and how this is reflected in the stable isotope ratios of materials that they produce. Isotopic analyses can thus be used to study life where direct observations are not possible. The authors also speculate on how the isotopic record would look if the biogeochemical C, N, and S cycles on Earth would operate in the absence of life. This comparison is important in the search for life on other planets. On Earth, by sequestering reduced carbon, nitrogen, and sulfur in minerals and recalcitrant kerogens, life separates electrons from their previous owners, which allows $\mathrm{O}_{2}$ to persist in Earth's present-day atmosphere. In the absence of life, the burial fluxes of organic matter, the composition of the atmosphere, and the oxidation state of the exosphere likely would have looked very different than today. Microbes would not have mediated a plethora of kinetically slow reactions, such as sulfate reduction or atmospheric $\mathrm{N}_{2}$ fixation, with important implications for the chemical and isotopic facets of geochemical cycles. 


\section{Future Missions Related to the Determination of the Elemental and Isotopic Composition of Earth, Moon and Planets}

Dandouras et al. review the contribution of future space missions to the determination of the elemental and isotopic composition of Earth, the Moon, the Solar System and exoplanets. Several planned missions to the Earth-Moon system will study the formation history of the Moon, the structure and dynamics of its exosphere, and its interaction with the radiation, solar wind and Earth's escaping atmosphere which might in the past has even been implanted into the Lunar surface. The authors also review the current plans for missions in near Earth orbit that will help better quantify atmospheric escape processes and Earth's upper atmosphere and magnetosphere. Future missions to Venus and Mars will further be key to establish a comparative view of the evolution of the climates and habitability of Earth, Venus, and Mars. The authors point out that sample return is the main task of future exploration, not only for these planets but also for comets and asteroids, which will give an insight into planetary building blocks. Returning samples from the atmosphere or surface of Venus would be the most challenging mission to fly but it would deliver a huge scientific return helping to solve the open questions on why Earth's sister planet is so different. Future missions to Mercury are further expected to provide a test bed for science of hot rocky exoplanets that orbit in close-in stellar environments. These authors conclude their review with a focus on the relevance of the spectroscopic detection of $\mathrm{N}_{2}-\mathrm{O}_{2}$-dominated atmospheres for the search of extraterrestrial life by future space observatories.

\section{Supplemental Information on Earth's $\mathbf{N}$ and C-Cycles and the Latest Collection of Relative Atomic Solar System Abundances, Atomic Masses and Solar Photosphere and Major Meteoritic Compositions}

For a better understanding how the N- and C-cycles work on Earth and most likely also on terrestrial exoplanets with comparable functioning carbon-silicate cycles, a Special Communication article by Halama and Bebout is also included in this topical collection that briefly reviews for an interdisciplinary readership how nitrogen and carbon are converted into the biogeochemical cycle by which these elements are exchanged among the biosphere, pedosphere, geosphere, hydrosphere, and atmosphere of the Earth.

Finally, the latest data for atomic abundances and mass fraction for the elemental and isotopic solar system composition, the atomic masses of the elements and their isotopes, the composition of the solar photosphere, and the compositions of the major chondritic meteorite groups is given in a second Special Communication by Lodders. The data which are relevant for all review articles and their interested readers are summarized in comprehensive tables that finish this topical collection of relevant review articles related to "Reading Terrestrial Planet Evolution in Isotopes and Element Measurements".

This collection is based on an interdisciplinary international workshop which took place at ISSI Bern during October 22 and 26, where about 48 leading scientists discussed the issues presented here.

Publisher's Note Springer Nature remains neutral with regard to jurisdictional claims in published maps and institutional affiliations. 